\title{
ELEMENTOS DE OSTEOSINTESIS DE USO HABITUAL EN FRACTURAS DEL ESQUELETO APENDICULAR: EVALUACION RADIOLOGICA
}

\author{
Drs. César García $M^{(1)}$, Dulia Ortega $T^{(2)}$.
}

1. Servicio de Radiología, Clínica Alemana de Santiago.

2. Centro de Imagenología, Hospital Clínico U. de Chile.

\begin{abstract}
Coping with orthopaedic hardware imaging has always been difficult for radiologists. We don't know adequately fixation devices implemented in fracture fixation, their characteristics, biomechanical principles, potential complications and how to report them properly. The following is a revision of these topics and suggestions in order to improve our skills in reporting as well.
\end{abstract}

Key words: Fractures, Grafts, Stents and prostheses.

Resumen: El abordaje radiológico de las imágenes del arsenal traumatológico ha sido siempre un tema de manejo difícil para los radiólogos, ya que no conocemos adecuadamente los diferentes aparatos de fijación utilizados, sus características, principios biomecánicos de aplicación, posibles complicaciones y cómo informarlas apropiadamente. Revisamos en este artículo estos puntos y hacemos algunas consideraciones para su informe.

Palabras claves: Fracturas, Injertos, Prótesis.

\section{Objetivo}

Revisar la evaluación del tratamiento de fracturas del esqueleto apendicular a través de una puesta al día de los elementos de fijación empleados, describiendo además los grupos genéricos utilizados para fijarlas y estabilizarlas. Daremos también algunas pautas para elaborar el informe radiológico.

\section{Introducción}

La evaluación de las intervenciones traumatológicas no es tarea fácil, dado que generalmente no conocemos el arsenal quirúrgico utilizado, sus indicaciones, principios biomecánicos y complicaciones derivadas de su uso. Es necesario, por

García C, y cols. Elementos de osteosíntesis de uso habitual en fracturas del esqueleto apendicular: Evaluación radiológica. Rev Chil Radiol 2005; 11: 58-70.

Correspondencia: Dr. César García M.

cgarcia@alemana.cl

dortega@ns.hospital.uchile.cl tanto, conocerlos, especialmente los usados en nuestros lugares de trabajo, y saber qué parámetros debemos evaluar para un mejor informe radiológico.

De las múltiples intervenciones sobre el hueso un común denominador son los elementos de osteosíntesis (OTS), aparatos mecánicos construidos principalmente de acero inoxidable, titanio o elementos biodegradables.

Centraremos nuestra revisión en las fracturas del esqueleto apendicular, que es la patología ósea de mayor frecuencia. Ellas pueden ser tratadas con: a) Métodos cerrados, en los que se hace reducción cerrada, estabilización e inmovilización con yeso y menos comúnmente con aparatos de tracción. b) Métodos abiertos, en los que la reducción es quirúrgica o percutánea, y se efectúa estabilización y fijación interna con aparatos de OTS. c) Métodos externos con reducción, estabilización y uso de fijadores externos.

Cada vez que es posible, la mayoría de los traumatólogos prefiere tratar con reducción cerrada, recuperación del alineamiento óseo y estabilización con aparatos de tracción o compresión externa, como valvas o yesos. En caso contrario, efectúan la fijación quirúrgica con reducción anatómica de los fragmentos y mantención con algún sistema de fijación, preservando el flujo sanguíneo al hueso y tejidos blandos, utilizando la técnica menos traumática posible para permitir una rápida cicatrización, recuperación precoz de la movilidad y función completa del miembro dañado.

La concepción actual, sin embargo, es buscar la reducción más fisiológica posible y no necesariamente un alineamiento perfecto.

\section{Elementos utilizados en el tratamiento cerrado u ortopédico.}

Su objetivo principal es promover la formación de callo externo, estimulándolo con recuperación precoz de la actividad muscular, del movimiento articular y transmisión de carga. La fractura se reduce, estabiliza e inmoviliza mediante maniobras externas al foco, sin alteración del flujo sanguíneo. 
La estabilidad mecánica se alcanza por lo general entre 6 y 18 semanas, después de lo cual la reparación ósea continúa por formación de callo intramedular y remodelación a lo largo de las líneas de estrés ${ }^{(1,2)}$. Es habitual que los métodos ortopédicos sean de recuperación más lenta en cuanto a liberación de movilidad y carga axial.

Los principales métodos de tratamientos cerrados de fracturas son los yesos de todo tipo y los aparatos de tracción.

Los yesos utilizan, para mantener las fracturas reducidas en posición, el principio del cilindro hidráulico en donde su forma y rigidez relativa mantienen la longitud y el alineamiento, funcionando como un cilindro que resiste el edema y la deformación (Figura 1).

El uso de tracción es limitado en la actualidad. Existen dos formas: la cutánea, que se aplicó en fracturas femorales en niños pequeños, prefiriéndose actualmente el enclavijado intramedular y la esquelética, prácticamente no utilizada ya que ha sido reemplazada por fijación externa o interna ${ }^{(3)}$.

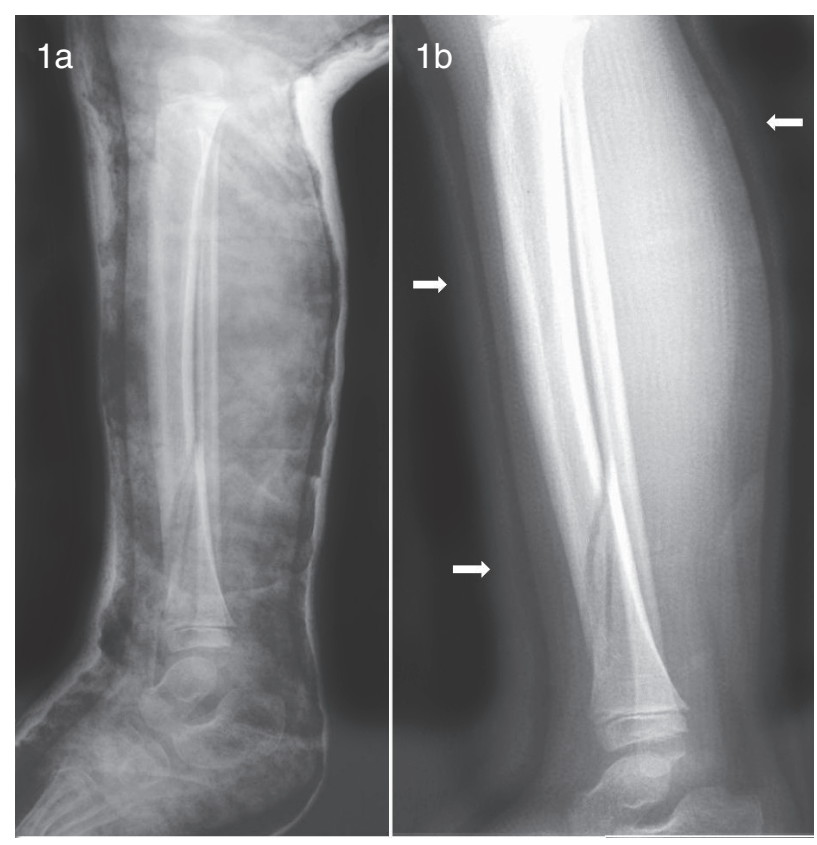

Figura 1 a, b. Yesos. Fractura de la diáfisis tibial. a) Yeso clásico. b) La misma paciente con un yeso plástico (flechas).

\section{Elementos de fijación externa}

Se usan en fracturas expuestas con pérdida de masa ósea o infección, en politraumatizados con compromiso de conciencia, elongación de extremidades y otros. Evita el trauma quirúrgico, preservando el flujo vascular ${ }^{(4,5)}$.

Consisten de alambres o agujas colocados en el hueso por encima y debajo del foco de fractura y unidos entre sí por barras verticales. Existen tres tipos: 1) El estándar uniplanar. 2) Fijadores híbridos, que son una combinación de dos uniplanares o un uniplanar y un anular fijados al hueso con púas transfixiantes, y dependen de los modelos disponibles en el mercado. 3) Fijador anular, del cual uno de los más conocidos es el Ilizarov, que forma una especie de exoesqueleto que circunda al hueso; está compuesto de marcos circulares fijos con alambres unidos entre sí por barras verticales. Funcionan sobre la base del principio biomecánico de la compresión externa y están diseñados para resistir el estrés cizallante y permitir la movilidad y carga fisiológica precoz estimulando el proceso de cicatrización secundario (Figura 2).

Las complicaciones de todos estos sistemas incluyen infección del trayecto de la púa, aflojamiento de éste, secuestros en anillo y rotación de los fragmentos $^{(4,6-8)}$.

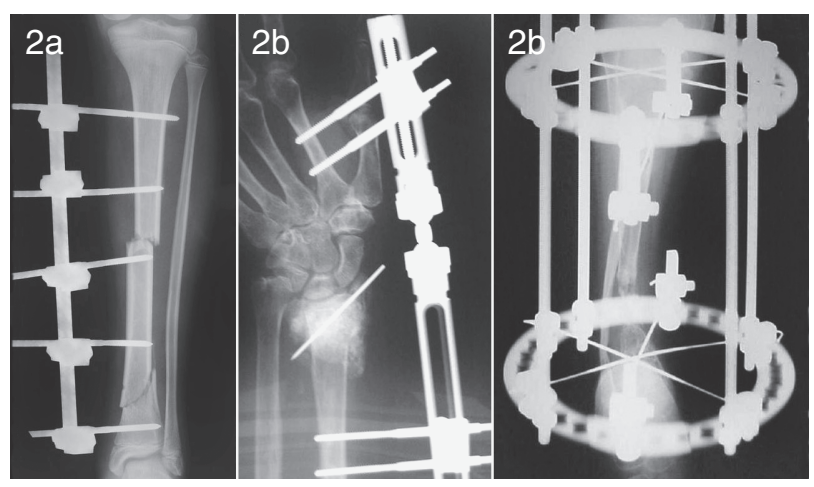

Figura 2 a-c. Fijadores externos. a) Uniplanar. b) Uniplanar articulado. c) Externo anular (Ilizarov) fijando una fractura infectada de la tibia (Imagen gentileza Dr. Aníbal Espinoza).

\section{Elementos de fijación interna}

La fijación interna requiere la exposición quirúrgica del foco de fractura, reducción abierta de los fragmentos y colocación de un elemento mecánico para mantener la reducción hasta la cicatrización completa. Tiene la ventaja de producir un alto grado de estabilidad mecánica, siendo su desventaja el trauma quirúrgico asociado.

La conformación rígida de la fijación limita el movimiento entre los fragmentos a tal grado que no se produce formación de callo óseo externo, por lo que se debe mantener por plazos mayores. Es necesario que el aparato constituya un todo con el hueso dañado para resistir el estrés de la actividad fisiológica. Actúan basados en los principios biomecánicos de la compresión fragmentaria, el puente y la inmovilización interna(4,5).

La compresión fragmentaria mantiene unidos mecánicamente los fragmentos y puede ser estática o dinámica. La primera adosa los fragmentos, no permite movimiento en el foco de fractura con la carga fisiológica y produce cicatrización primaria, que en las radiografías se ve como una desaparición 
gradual de la línea de fractura. Tornillos y placas corticales son buenos ejemplos. En la compresión dinámica el fijador transforma la carga fisiológica en compresión a nivel del foco de fractura. Así actúan bandas de tensión, placas de contención o sostén, tornillos dinámicos de cadera y clavos intramedulares no bloqueados.

Un elemento de fijación interna actúa como puente cuando se ancla en hueso sano proximal y distal al foco de fractura, facilitando la transmisión de la carga fisiológica del hueso proximal al hueso distal, sin pasar por el foco de fractura. Como los fragmentos no están directamente adosados hay movimiento que se traduce en cicatrización endóstica y perióstica. Los clavos intramedulares actúan con frecuencia de este modo(1).

Los innumerables elementos de fijación interna se pueden dividir en unos pocos grupos: A) Golillas y grapas. B) Púas y agujas. C) Alambres. D) Tornillos. E) Placas. F) Clavos intramedulares.

A. Golillas y Grapas: Se utilizan ocasionalmente en osteotomías, artrodesis o fijación de fracturas. Las golillas planas incrementan la superficie del área sobre la que se distribuye la fuerza ejercida por el tornillo de fijación, previniendo la rotura de la cortical bajo su cabeza; las aserradas tienen bordes espiculados y se utilizan principalmente en la fijación de tendones avulsionados. Las grapas se utilizan mayoritariamente en fijación de artrodesis, en epifisiodesis del esqueleto inmaduro y fijación de osteotomías correctoras $^{(5,9)}$ (Figura 3).

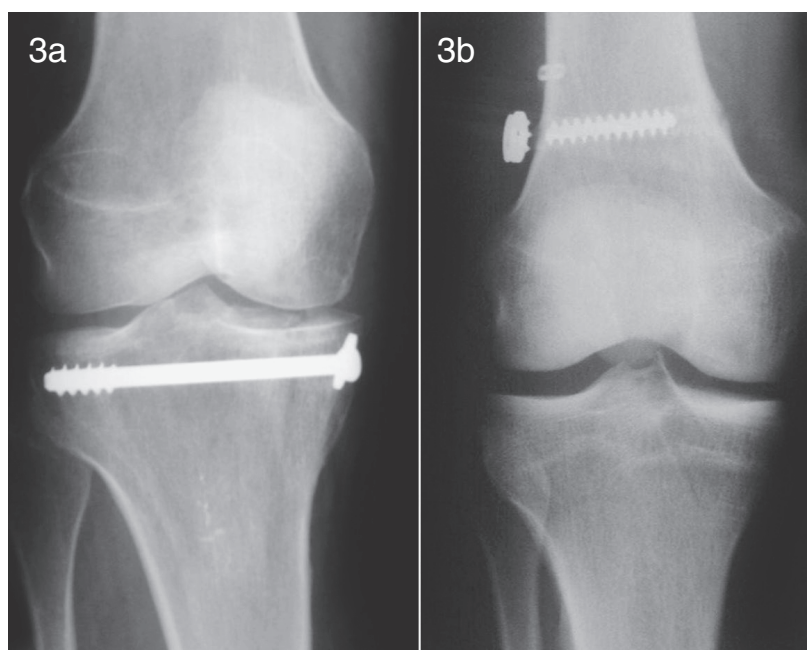

Figura 3 a-b. Golillas. a) Golilla plana en estabilización de fractura tibial. b) Golilla aserrada de bordes espiculados.

B. Agujas: Existe una amplia gama de variados tamaños, con o sin rosca. Las más utilizadas, solas o en combinación, son las agujas de Kirschner $(\mathrm{K})$ que pueden ser usadas como guías para la introducción de tornillos canulados, en aparatos de tracción, fijadores externos y como fijadores transitorios o permanentes de fracturas. Son

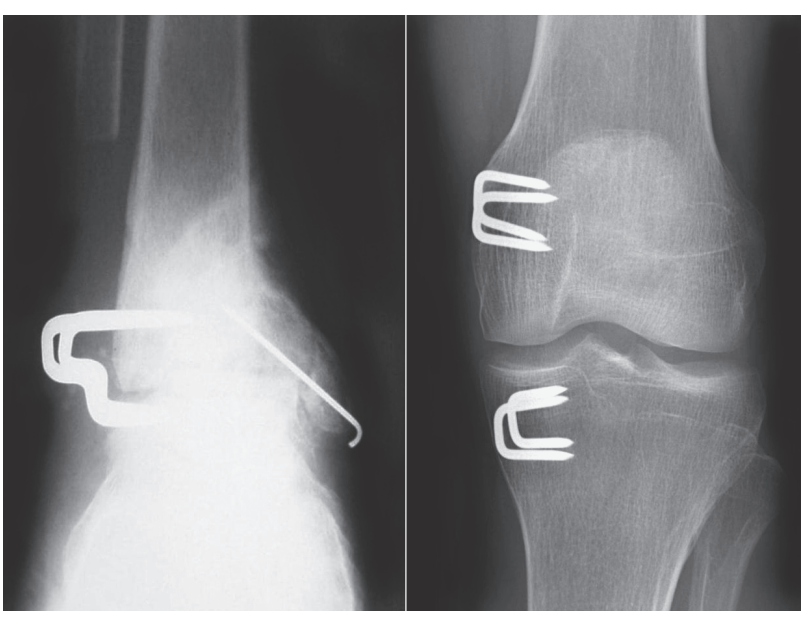

Figura 3 c-d. Grapas. c) Tobillo AP. Grapa Coventry estabilizando una artrodesis por el lado peroneo. Fijación con aguja K por el lado tibial. d) Grapa fijando plastía de ligamento cruzado anterior en el túnel tibial.

las más delgadas, tienen puntas espatuladas y son lisas. Las púas de Steinmann son más gruesas y tienen rosca parcial. Sus usos son similares a los de las agujas $\mathrm{K}^{(5,10)}$ (Figura 4).

C. Alambres: Existe una gran variedad, siendo utilizados como elemento único, o con mayor frecuencia en combinación con otros elementos de fijación. Hay dos tipos principales que comprenden los cerclajes y las bandas de tensión ${ }^{(1,5)}$.

Los cerclajes con alambres o cables se colocan en forma circunferencial. Se suelen utilizar en conjunto con fijación endomedular o en combinación con prótesis de cadera. Esta técnica libera al hueso de las fuerzas tensionales en el foco de fractura, comprimiéndolo. Su complicación potencial es la interrupción del flujo sanguíneo periostal, con necrosis ósea o ausencia de consolidación secundaria (Figura 5a).

Las bandas de tensión son utilizadas solas o en combinación con tornillos o agujas K. Su principio de acción se basa en utilizar la fuerza muscular que normalmente tracciona hacia proximal el hueso afectado, para aplicar compresión fragmentaria. Indicadas principalmente en fracturas de rótula o del olécranon ${ }^{(1,5)}(5 \mathrm{~b}, \mathrm{c})$.

D. Tornillos: Son uno de los elementos de OTS más utilizados. Existe una gran variedad y podemos agruparlos en dos tipos básicos: los de cortical y los de esponjosa (Figura 6). Los primeros tienen rosca o hilo en toda su longitud; su principal indicación es la fijación de placas al hueso y se utilizan en la diáfisis yendo de cortical a cortical. Los de esponjosa están diseñados para atravesar segmentos largos de hueso esponjoso, por lo que tienen hilo o rosca más espaciado y grueso sólo en su parte distal. Ambos tipos de tornillos reciben distintos nombres de acuerdo a su función o morfología ${ }^{(1,4)}$. 


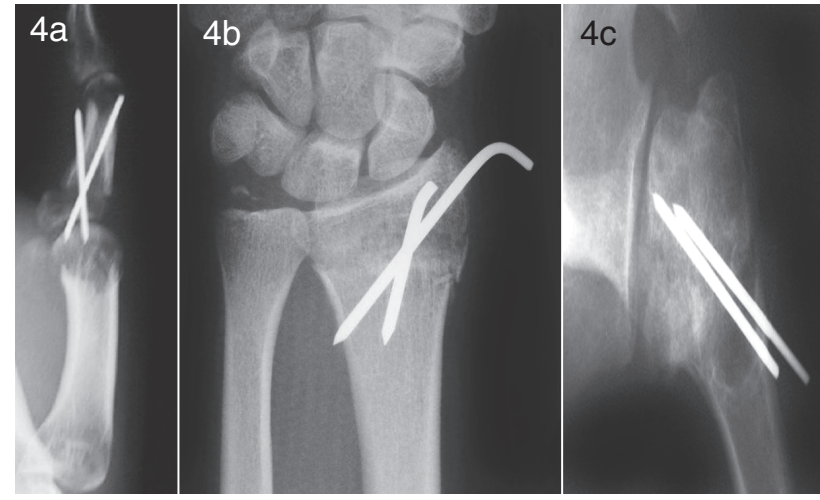

Figura 4 a-c. Agujas: a) Agujas $K$ en fractura de la falange proximal del pulgar. b) Agujas $K$ en fractura conminuta de la epífisis distal del radio. c) Agujas Steinmann en húmero proximal.

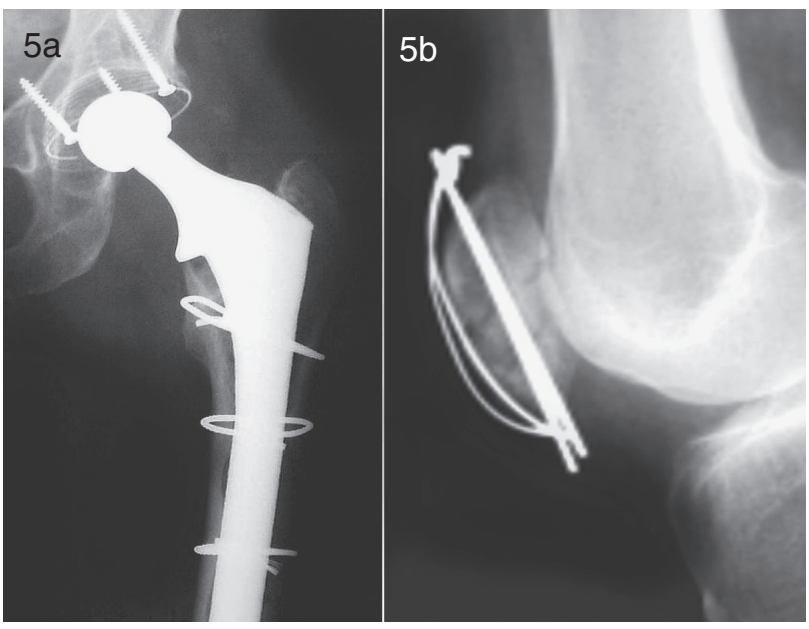

Figura 5 a-c. Alambres: a) Cadera AP. Prótesis total no cementada, asegurada con tres bandas de cerclaje. b) Rótula. Fractura fijada con banda de tensión y agujas $K$.

D1. Tornillo de compresión fragmentaria. Estos comprimen dos objetos, como hueso contra hueso o hueso contra otro elemento de fijación. Tienen rosca distal que se inserta completamente en el hueso alejado de la fractura, traccionándolo hacia el fragmento proximal que ha sido atravesado por el trozo sin rosca. Puede ser colocado a través del agujero de una placa.

Un tornillo con rosca completa, cuya extracción es más fácil, fija dos objetos contiguos sin comprimirlos porque los hilos del tornillo los obligan a mantener sus posiciones relativas. Sin embargo, pueden ser utilizados para compresión.

Los tornillos fragmentarios están indicados en la fijación de fracturas articulares y yuxta-articulares para conseguir reducción anatómica y adecuada estabilidad $^{(1,4-5)}$ (Figura 7 a).

D2. Tornillos sindesmóticos. Son estabilizadores de la articulación tibio-peronea distal. Se

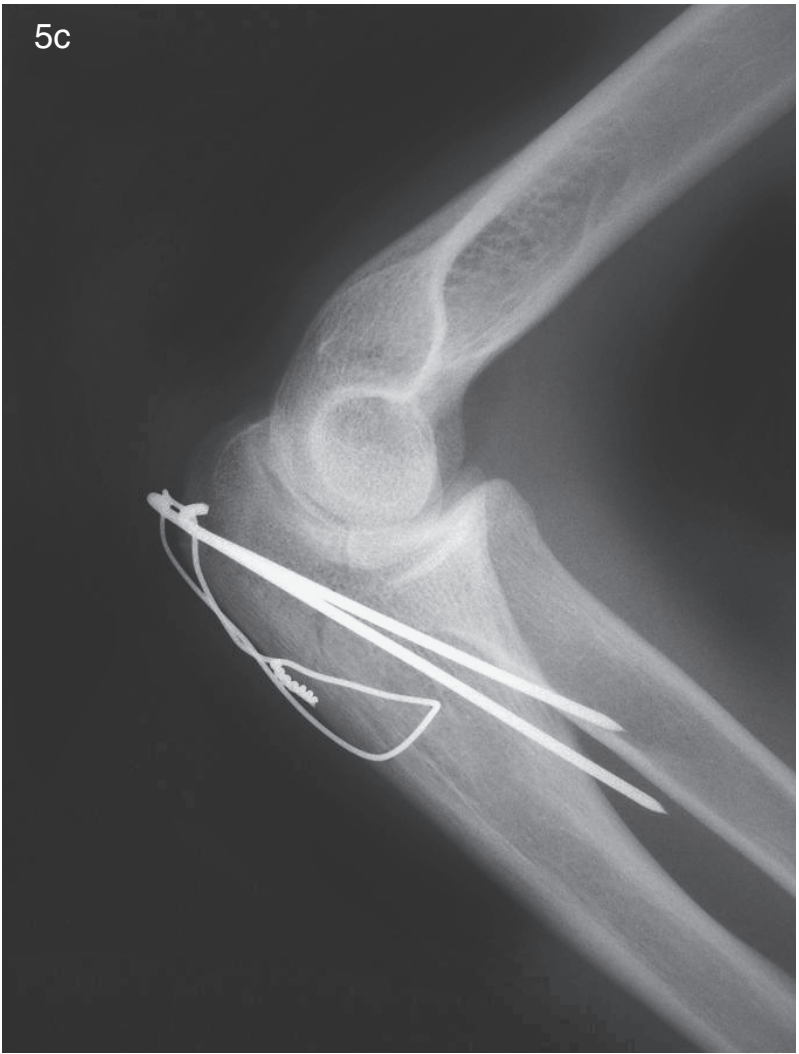

Figura 5 c. Alambres: c) Codo. Fractura de la base del olécranon fijada con banda de tensión y aguja $K$.

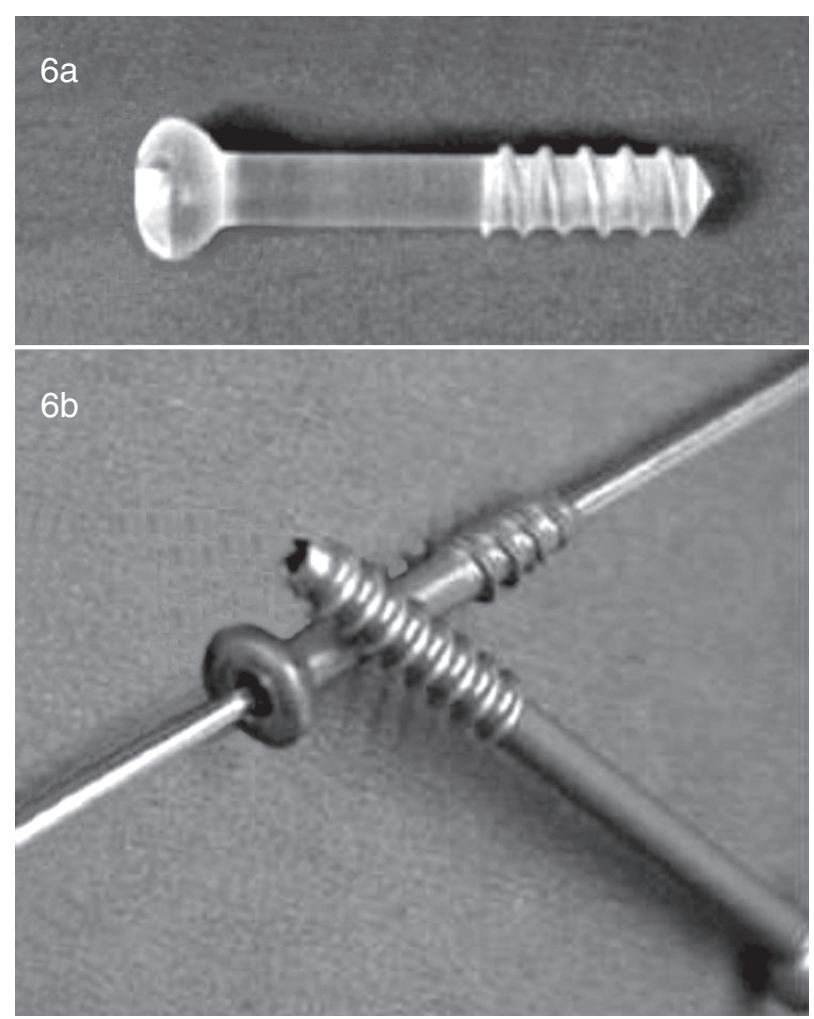

Figura 6 a-b. Algunos tipos de tornillos: a) Tornillo biodegradable de esponjosa (Stealth Corp. USA). b) Tornillo de esponjosa canulado. 
colocan en forma paralela $102 \mathrm{~cm}$ sobre la superficie articular del tobillo. También puede ser colocada a través de un agujero de una placa de fijación del peroné (Figura $7 \mathrm{~b}$ ).

D3. Tornillos canulados. Son huecos, permiten una colocación más exacta a través de alambres guías. Cuando son usados para tratar fracturas subcapitales del cuello femoral se ponen tres paralelos, vía percutánea con guía de alambre y apoyo fluoroscópico ${ }^{(1,4)}$ (Figura 7 c,d).

D4. Los tornillos de diseño especial. Son utilizados en ciertas regiones anatómicas específicas.

El de Herbert se utiliza preferentemente en fracturas de escafoides; es canulado, posee roscas de distinto tamaño en sus dos extremos, es liso en el centro y no tiene cabeza. Actúa produciendo compresión de los dos fragmentos entre sí, debido al diferente tamaño de las roscas de ambos extremos (Figura $7 \mathrm{e}$ ).

El Acutrak también se usa para tratar fracturas de escafoides, es canulado, sin cabeza, lo que permite su localización intraósea; utiliza el mismo concepto de las roscas diferentes en ambos extremos, pero el hilado es completo, lo que aumenta la capacidad de compresión ${ }^{(2,5)}$ (Figura 7 f).

D5. Los tornillos de anclaje. Más conocidos como anclas metálicas, están diseñados para servir como medios de sujeción de ligamentos, tendones o cápsulas dañadas. Su morfología incluye puntas y ganchos para una mejor fijación al hueso o los tejidos blandos, y facilitar la fijación de los ligamentos al elemento de anclaje y hueso. Pueden ser metálicos o biodegradables ${ }^{(4,8)}$ (Figura 8).

D6. Tornillos de interferencia. Utilizados en la reconstrucción del ligamento cruzado anterior (LCA). Hay una amplia gama, pero todos tienen por función actuar como elementos de anclaje y fijar los injertos en los túneles óseos femorales y tibiales construidos para tal efecto. Estos tornillos comprimen el injerto contra la pared lateral del túnel, evitando su deslizamiento. Son cortos, anchos, con rosca completa alta y ancha, con cabeza embutida. Pueden ser metálicos o bioabsorbibles ${ }^{(1,5,11-14)}$ (Figura 9 a-f)

D7. El tornillo de compresión dinámica o tornillo dinámico de cadera (Dynamic Hip Screw o $D H S)$ se utiliza en el tratamiento de fracturas intertrocanterianas o pertrocantéreas. Conformado por un tornillo de tracción de gran diámetro, con rosca en su extremo, debe quedar centrado en la cabeza femoral. Va unido a una placa lateral que se fija al hueso con tornillos corticales y que termina en un cilindro hueco donde se aloja el tornillo, que se desliza por el cilindro a medida que se produce la reabsorción ósea, y por la carga del paciente al caminar precozmente. Actúa comprimiendo los fragmentos en el sitio de fractura durante el proceso
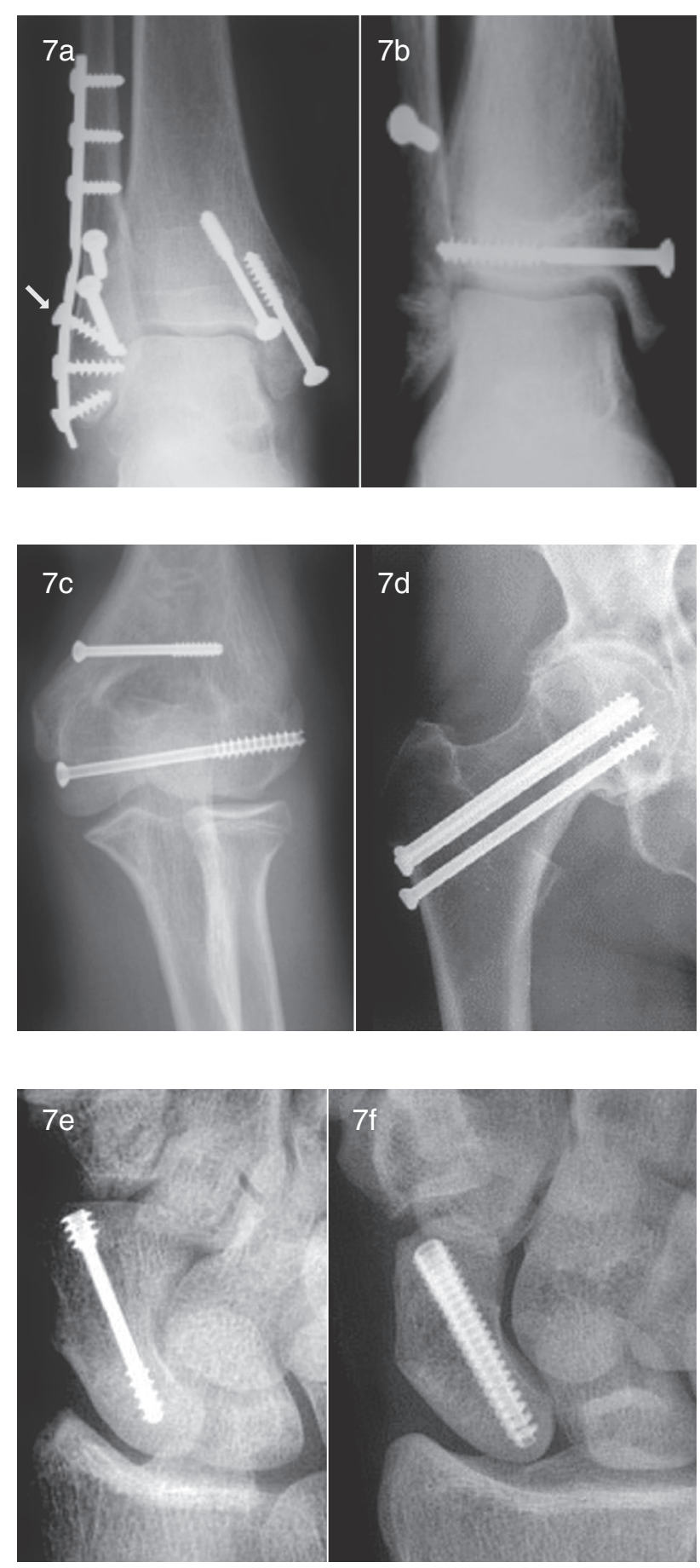

Figura 7 a-f. Tornillos: a) Tobillo AP con 2 tornillos fragmentarios con rosca distal en fractura de maléolo tibial y 2 en fractura de peroné. Placa de compresión dinámica fija al peroné con tornillos corticales, uno de ellos (flecha) utilizado como fragmentario. b) Tornillo sindesmótico, de rosca parcial en articulación tibio-peronea distal y tornillo fragmentario de rosca completa uniendo fragmentos de fractura peronea distal. c) Codo AP. Tornillos canulados de esponjosa en fractura de paleta humeral. d) Cadera AP. Hay 3 tornillos de esponjosa canulados que fijan una fractura subcapital del cuello femoral.

Tornillos utilizados en fracturas de escafoides. e) Tornillo de Herbert, con rosca en ambos extremos. f) Tornillo Acutrak, con rosca completa, de hilado diferente en cada extremo. 

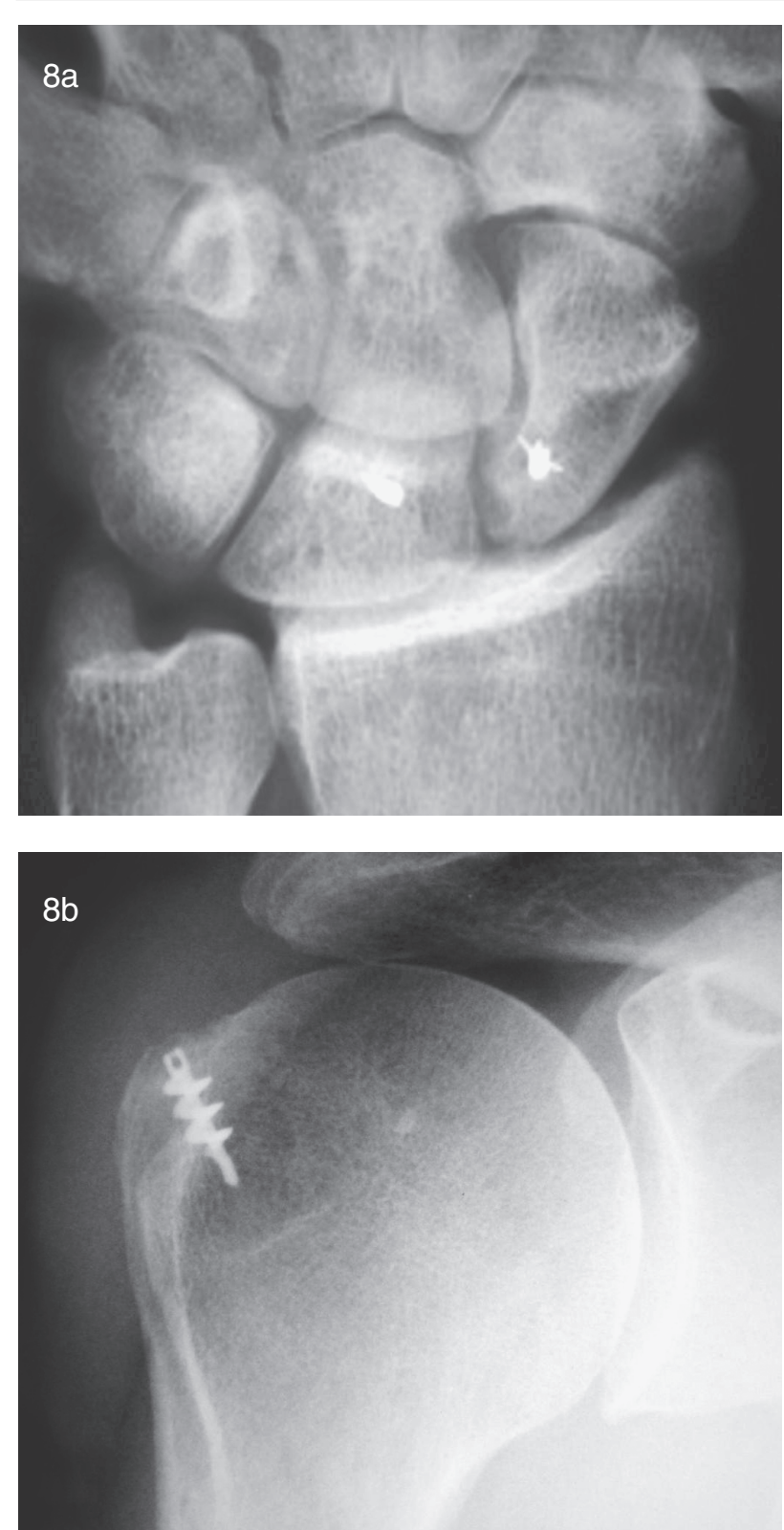

Figura 8 a,b. Elementos de anclaje: a) Anclas en escafoides y semilunar para reparación ligamentosa b) Hombro AP. Elemento de anclaje en troquíter para fijación del tendón del músculo supraespinoso.

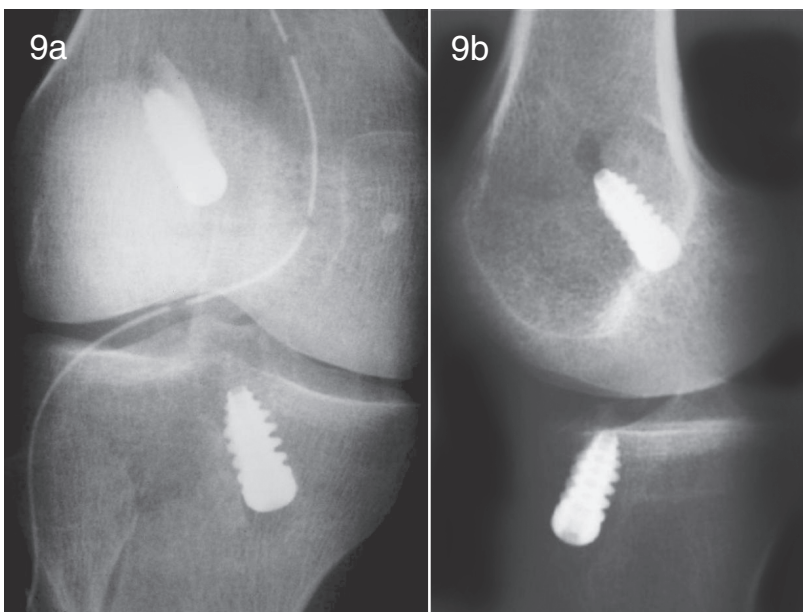

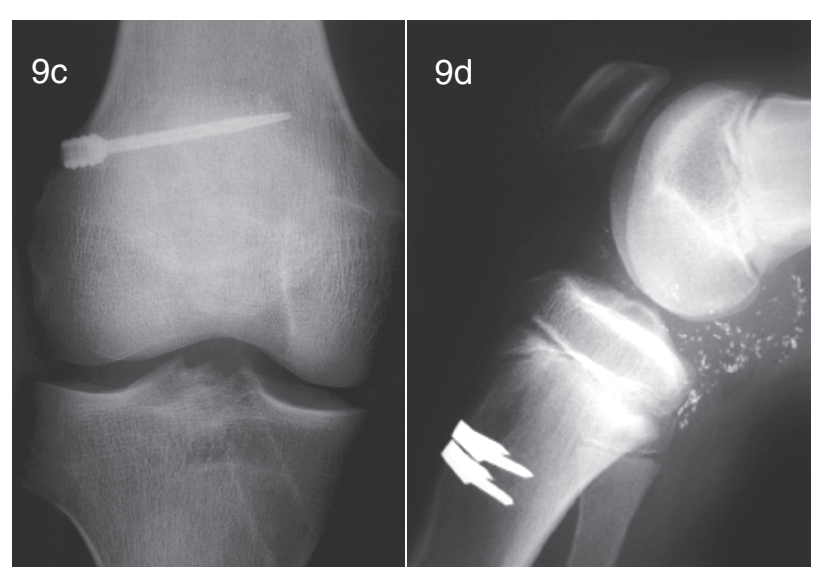

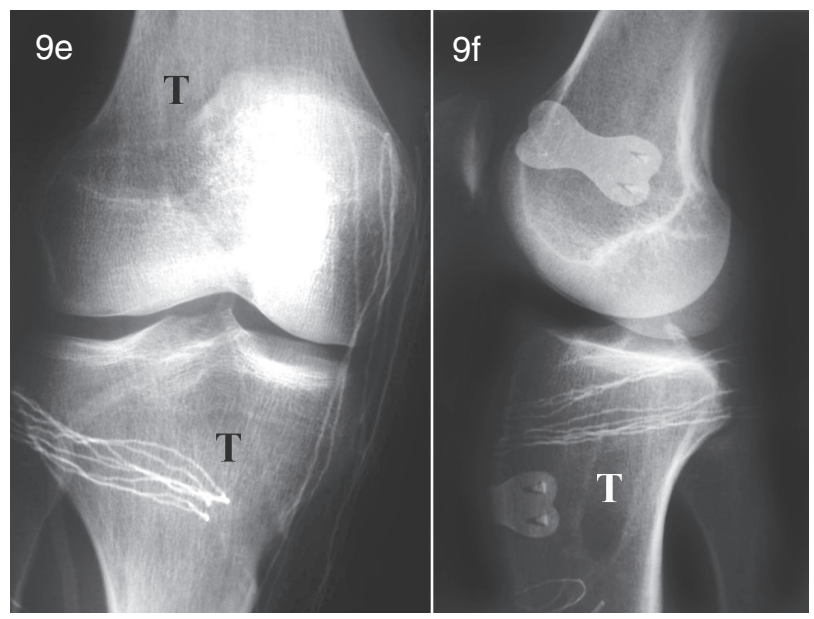

Figura 9 a-f. Tornillos de interferencia en rodilla: $a, b)$ Tornillos ubicados en túneles tibial y femoral. c) Proyección AP con púa de sujeción del injerto de LCA en túnel femoral y tornillo radiolúcido bioabsorbible en túnel tibial. d) Rodilla L con grapa de amarre del injerto de LCA en túnel tibial. Fragmentos metálicos. e,f) Reconstrucción de LCA con tornillos biodegradables radiolúcidos. Sólo se ven los túneles tibial y femoral (T).

de consolidación al soportar la carga fisiológica debido a que la cabeza y cuello femorales quedan en un ángulo anatómico, resisten la deformidad angular y permiten la impactación precoz de la fractura. El grado de telescopaje observado en las radiografías de seguimiento es la prueba de esta mayor impactación ${ }^{(1,5,15)}$ (Figura 10).

D8. Los tornillos de compresión dinámica (TCD) condilares. Desarrollados para el tratamiento de fracturas de la región supracondílea, se utilizan también en algunos casos en fracturas subtrocanterianas. Están diseñados según los principios del DHS, son más gruesos y resistentes, y el ángulo placa-cilindro tiende a ser recto(1,16-18) (Figura 11).

E. Placas: Existen diferentes modelos, fabricadas de acero inoxidable o de titanio, tienen varios agujeros y se colocan sobre la superficie de los huesos, aseguradas con tornillos. No necesa- 


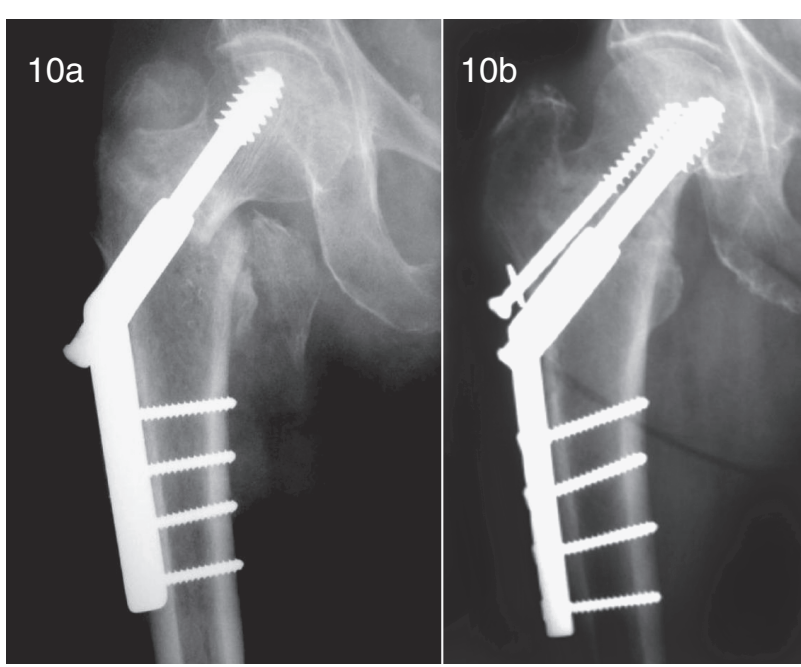

Figura 10 a, b. Tornillo de compresión dinámica de cadera (DHS): a) Tornillo utilizado para fijar fractura transtrocanteriana. b) Fractura de la base del cuello con un DHS y un tornillo fragmentario.

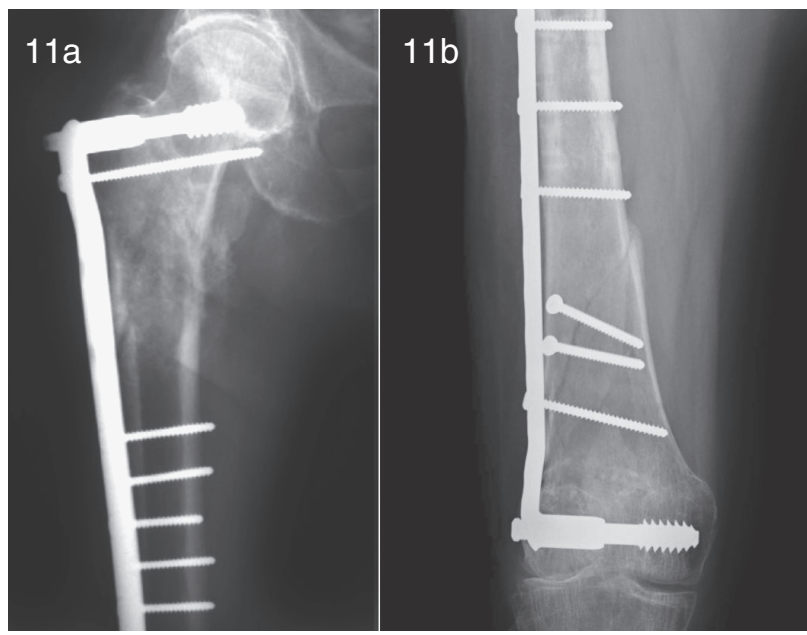

Figura 11 a, b. TCD condilares: a) Fémur proximal $A P$ con un TCD utilizado en fractura subtrocantérea. b) Fémur distal AP con un TCD, que fija fractura supracondílea.

riamente todos los agujeros son usados. Se clasifican sobre la base de algunos de sus atributos, ya sea forma, diseño de los agujeros, sitio elegido para la fijación o modo de aplicación.

Se utilizan con mayor frecuencia en fracturas de huesos largos, pero también en artrodesis de columna y muñeca. Requieren una incisión quirúrgica más amplia que otros tipos de fijación. Existe la posibilidad de alteración del flujo sanguíneo cortical, debido a la gran superficie de contacto, y de la consolidación, pudiendo reproducirse la fractura al retirar la placa, por atrofia ósea. En general, basan su funcionamiento en tres principios biomecánicos: compresión dinámica, neutralización y contención o sostén ${ }^{(4,5)}$. Existen también algunas de diseño especial.
E1. Las placas de compresión comprimen los extremos de la fractura, se usan para fijar fracturas estables manteniendo la reducción y compresión. La compresión también se puede alcanzar a través de agujeros de diseño especial o por medio de la colocación excéntrica de los tornillos. Se pueden utilizar conjuntamente con tornillos fragmentarios.

Las placas de compresión dinámica (PCD), diseñadas para la compresión axial, son uno de los tipos más utilizados; se reconocen por sus agujeros ovalados para la inserción excéntrica de los tornillos, cuyas paredes son biseladas hacia el piso e inclinadas hacia medial. La zona más débil de estas placas está alrededor de los agujeros ya que es la única zona que se puede doblar ${ }^{(1,4,5)}$ (Figura $12 \mathrm{a}, \mathrm{b}$ ).

La placa de compresión dinámica de bajo contacto o impacto (LCP) es un tipo de placa de compresión de desarrollo reciente, que se diferencia de la PCD por la forma del corte en su superficie inferior, que disminuye la superficie de aposición alrededor de los agujeros de los tornillos y entre éstos, minimizando la compresión placa-periostio, permitiendo mayor flujo capilar, y ayudando al proceso de cicatrización. Tiene cierto grado de deformación suave y elástica, sin concentrar el estrés alrededor de los agujeros ${ }^{(4)}$ (Figura 12c).

Las placas de reconstrucción maleables son de amplia utilización en fracturas de pelvis, calcáneo y codo. Son arrosariadas por los sacabocados entre los agujeros ovalados, lo que las hace maleables con facilidad en los tres planos, adaptándose a la forma y longitud requeridas en las superficies óseas complejas $^{(1,4)}$ (Figura $12 \mathrm{~d}$ ).

E2. Las placas de neutralización se colocan sobre un foco de fractura conminuta. Diseñadas para proteger la superficie de la fractura, transmiten las fuerzas de incurvación, torsión y carga axial. Con frecuencia se combinan con tornillos fragmentarios.

E3. Las placas de contención o sostén se usan en fracturas inestables como soporte del hueso delgado cortical periarticular, frente a las fuerzas de compresión o de carga axial, impidiendo su colapso. Se utilizan en radio distal y platillos tibiales (Figura 13).

E4. Placas de diseño especial. La placa «blade» se utiliza en fracturas supracondíleas. Tiene uno de sus extremos con ángulos cercanos a los $90^{\circ}$ para adaptarse a las variaciones individuales y con forma de cincel para ser introducido dentro de la metafisis. La placa se fija a la cortical con tornillos ${ }^{(1)}$ (Figura 14).

Las placas de diseño anatómico especial son una amplia gama, diseñadas para fines específicos: la placa condilar $95^{\circ}$ para estabilización de fracturas del fémur proximal y distal; la placa condilar de sostén o contención para fémur distal; placa T 4.5 para húmero y tibia proximales; placa T oblicua angulada 3.5 para radio distal, etc. ${ }^{(1)}$ (Figura 15). 

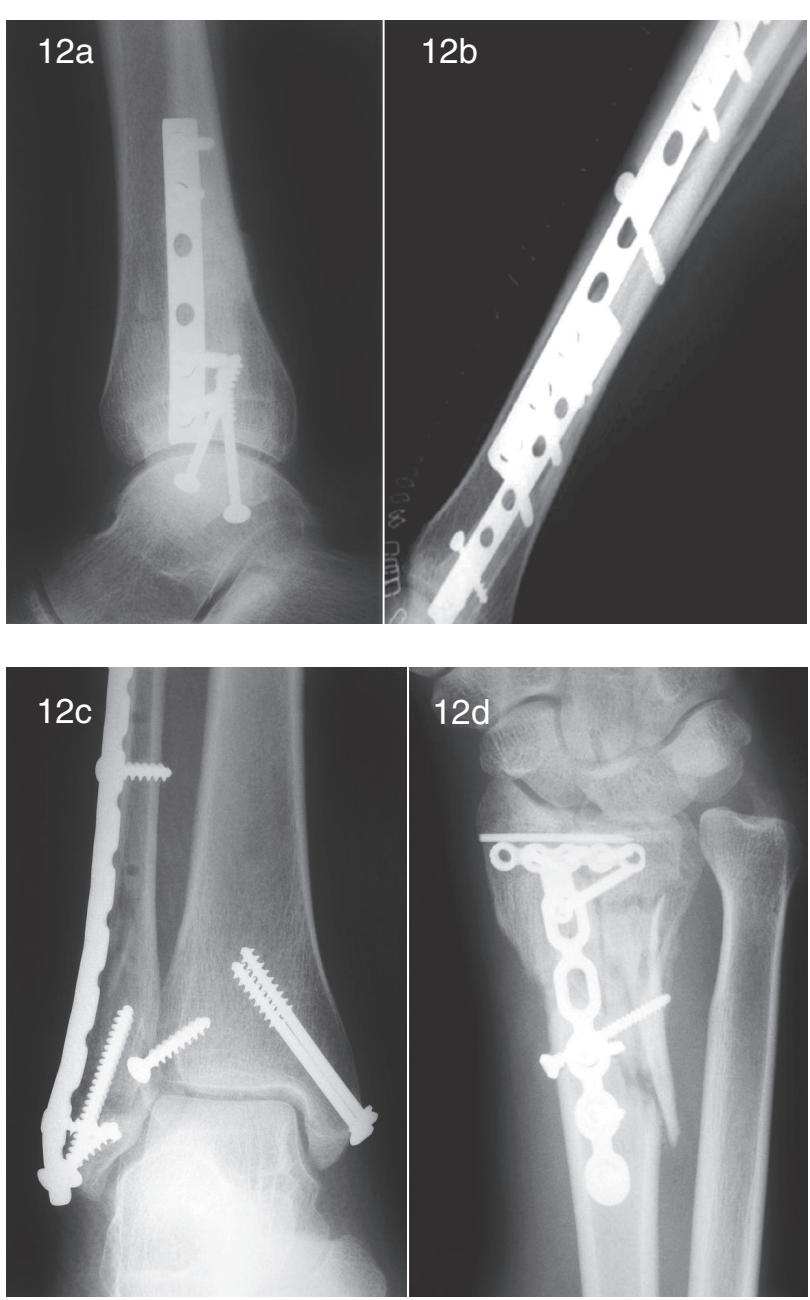

Figura 12 a, d. Placas. a,b) DCP que fija una fractura del peroné distal. Hay 2 tornillos fijando una fractura del maléolo tibial. Los agujeros son ovalados. c) DCP de bajo impacto diseñada para disminuir el área de contacto con el periostio, tiene muescas entre los agujeros de los tornillos. Tobillo AP fijación de peroné con dos tornillos bicorticales. Además se ven tornillos fragmentarios en maléolo tibial y uno de sindesmosis. d) Placa de reconstrucción. Muñeca AP demostrando una fractura conminuta de radio reducida y fijada con placa de reconstrucción, tornillo fragmentario y aguja.

\section{Placas con tornillos bloqueados o sistemas}

de fijación interna. Las placas de diseño convencional, como las ya descritas, siguen siendo de gran utilidad en el tratamiento de fracturas. Sin embargo, se está investigando para el desarrollo de técnicas que utilicen tanto placas como cirugías más biológicas, tratando de minimizar el daño de los tejidos blandos, reducir el uso de injertos y mejorar las tasas de cicatrización. Son las llamadas placas con tornillos bloqueados o sistemas de fijación interna, donde el hilo de la cabeza del tornillo y del agujero de la placa queda en un ángulo fijo dentro de la placa, impidiendo su deslizamiento (Figura 16 a). Actúan de modo similar a los fijadores externos. Se

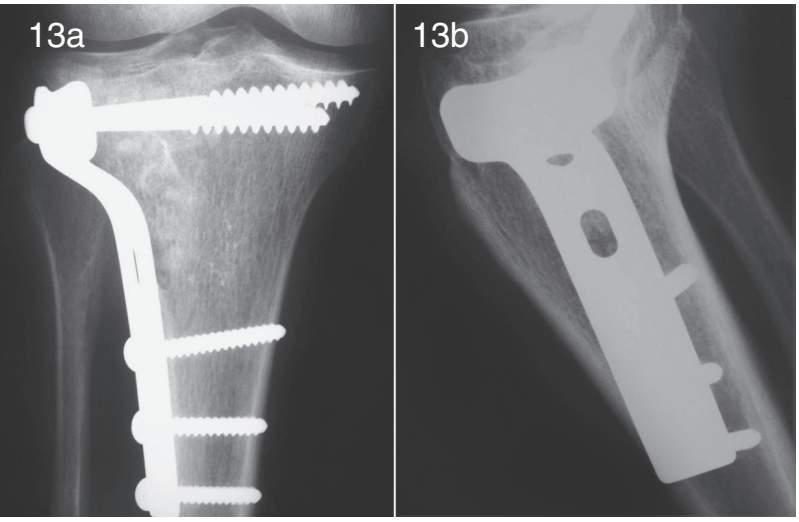

Figura 13 a,b. Placa de contención. Rodilla AP y L. Fractura de platillo tibial fijada con placa de contención que usa dos tornillos de esponjosa proximales y tres corticales. Nótese la forma del extremo superior de la placa que «sostiene» la meseta, manteniendo la congruencia articular.

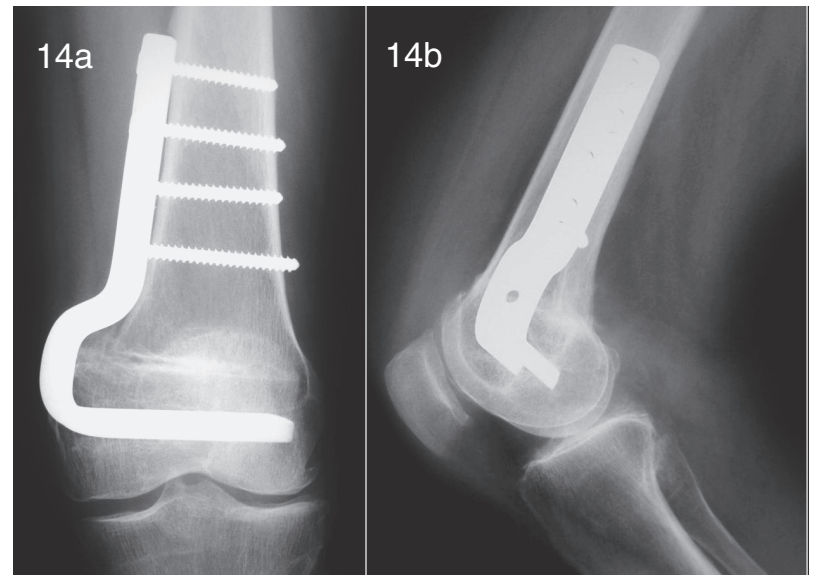

Figura 14 a,b. Placa «blade». Rodilla AP. Osteotomía correctora de ejes estabilizada con una placa «blade», con un extremo insertado en el área condilar y la placa lateral fija a la diáfisis con tornillos corticales.

implantan por medio de cirugías mínimamente invasivas, con accesos percutáneos e introducción submuscular. Están indicadas en fracturas periarticulares con metafisis muy conminutas. Son estudios preliminares y su validación está en curso $^{(4,18-20)}$.

Se han desarrollado dos grupos principales de placas con tornillos bloqueados. El fijador con puntos de contacto o PC-Fix es una placa estrecha, de superficie interna con pequeños puntos elevados para contactarse con la superficie ósea. Se fija al hueso con tornillos unicorticales auto-enroscantes. Diseñada para fracturas de los huesos del antebrazo, se están estudiando modelos para su uso en 


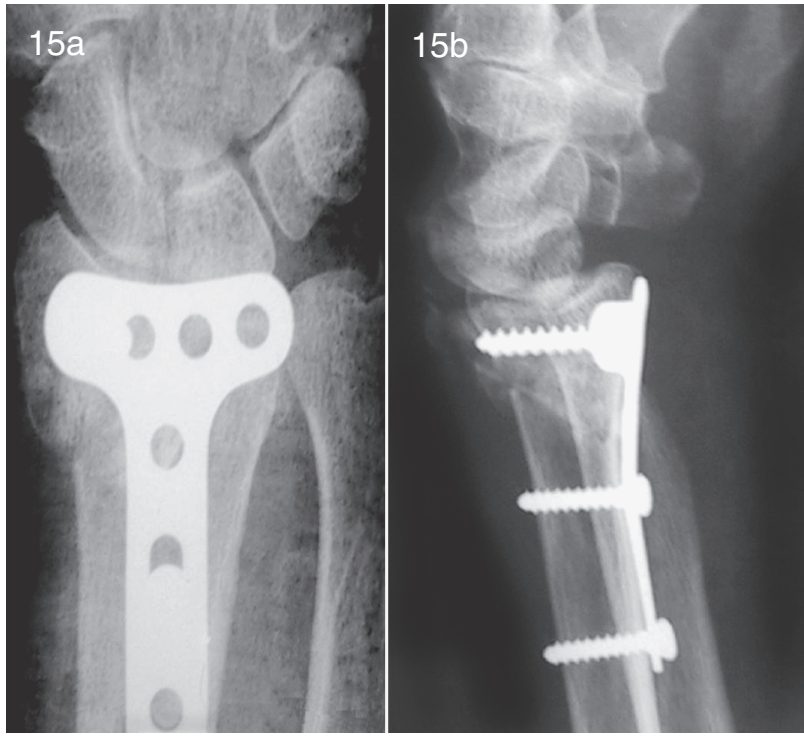

Figura 15 a,b. Placa de diseño especial. Muñeca AP y L. Fractura radial con extensión articular, estabilizada con placa $T$ angulada para radio distal.

fracturas de tibia y húmero(21). El sistema de estabilización menos invasivo (LISS) son placas que se ajustan al contorno anatómico de un área específica, con insertos distintos para lados derecho e izquierdo. No necesitan quedar en contacto con el hueso, por lo que no son maleables. Se fija al hueso con tornillos unicorticales bloqueados, y colocada a través de un acceso submuscular mínimamente invasivo. Los sistemas de fijación interna tienen evidentes ventajas sobre las PCD; preservación adecuada del flujo sanguíneo óseo, mayor resistencia a la infección, menos trauma quirúrgico ${ }^{(4,22,23)}$ (Figura $16 \mathrm{~b})$.

F. Clavos intramedulares: Hay una gran variedad, diseñados para colocación intramedular en huesos largos. Su diseño y técnica de inserción han evolucionado desde su introducción en la década de 1940. Son utilizados en la fijación de fracturas de la zona media de la diáfisis de fémur, tibia y húmero. La mayoría se coloca con técnica cerrada y mínima exposición de tejidos blandos, por vía anterógrada o retrógrada. Basan su funcionamiento en el principio biomecánico de la inmovilización actuando como puente en fracturas muy conminutas con gran compromiso de tejidos blandos, o en fracturas muy inestables. La consolidación se produce por la formación de callo perióstico y al existir cierto grado de movimiento se reduce el retardo o ausencia de ésta ${ }^{(1,5)}$.

Se dividen en rígidos y flexibles; los primeros de gran tamaño, en su mayoría son huecos para facilitar su introducción por medio de guías necesitan, antes de su colocación, ampliar la cavidad medular a medida que avanza. Dan excelente estabilidad frente
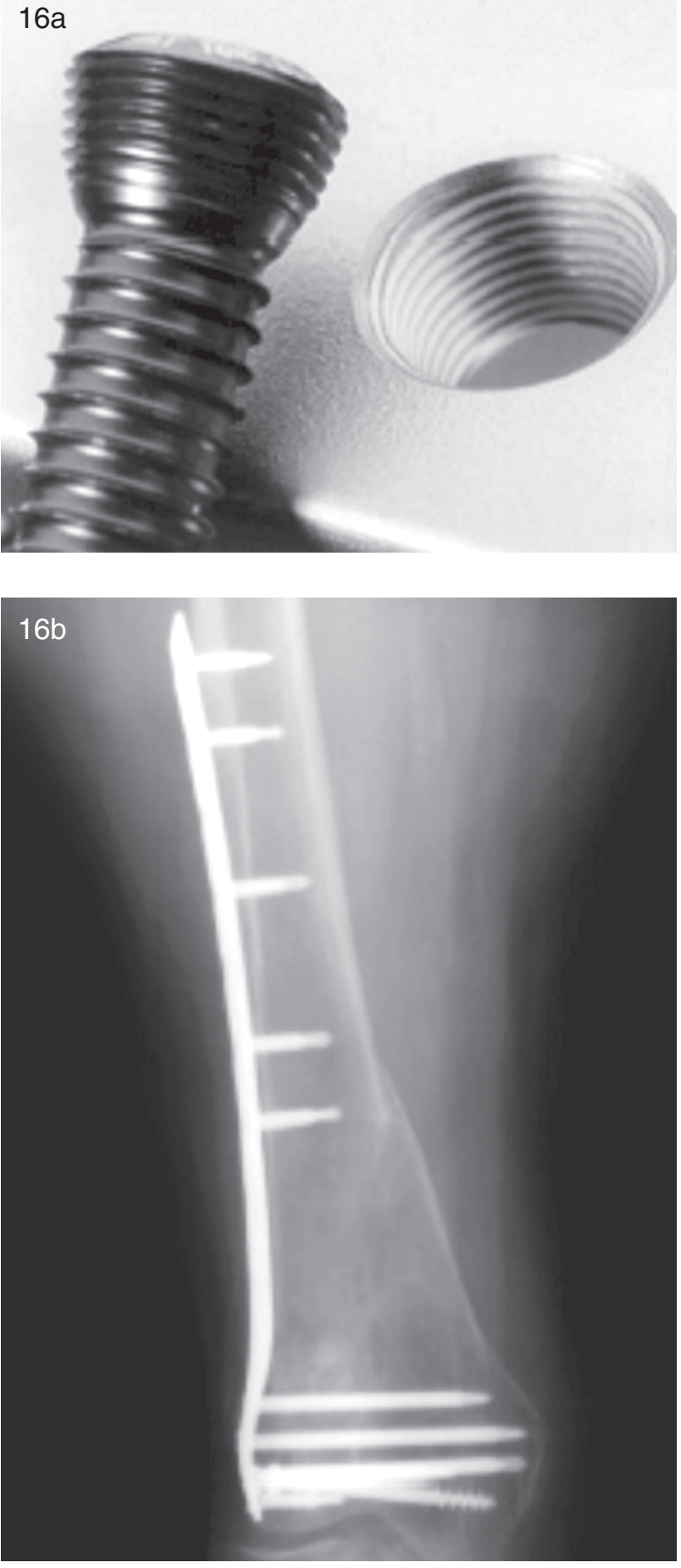

Figura 16 a,b. Placa con tornillos bloqueados: a) Detalle mostrando el hilo del agujero de la placa y la cabeza del tornillo que impiden el deslizamiento, creando un tornillo bloqueado en ángulo fijo. b) Fémur distal AP. Fractura conminuta supracondílea tratada con placa LISS con tornillos bloqueados unicorticales. Tornillos fragmentarios condilares.

a la incurvación, pero no ante las fuerzas rotatorias o de compresión, por lo que se añaden tornillos de bloqueo proximales, distales o ambos, que permiten soportar carga en forma precoz. Si el clavo está 
bloqueado a proximal y distal se dice que el bloqueo es estático porque todos los planos de movimiento están suprimidos; si, por el contrario, los tornillos están en un solo extremo, lo que permite cierto movimiento en el sitio de fractura el clavo está dinámicamente bloqueado(4, 24-26) (Figura 17).

Un tipo de clavo intramedular es el de reconstrucción diseñado especialmente para uso en fracturas de la diáfisis femoral asociadas a fracturas de cuello, intertrocanterianas o subtrocanterianas, sitio en el que el DHS no funciona adecuadamente por razones biomecánicas. Son mucho más resistentes y más invasivos que el DHS. Poseen agujeros proximales orientados de modo que los tornillos superiores se ajusten a la anatomía espacial de cabeza y cuello femorales(4,5) (Figura 18).

Los riesgos asociados al ensanchamiento intramedular, como el compromiso del flujo sanguíneo del endostio, osteonecrosis en la cara interna de la cortical, daño de tejidos blandos y la posibilidad de potenciales embolías grasas, llevaron al desarrollo de los clavos intramedulares flexibles. De diámetro pequeño, sólidos, muy flexibles, que se acomodan a la anatomía ósea, utilizándose en fracturas diafisiarias de los huesos largos del esqueleto inmaduro, insertados bajo guía fluoroscópica, se ponen varios en forma divergente hacia proximal o distal, a través de agujeros corticales en la metáfisis para no atravesar el cartílago de crecimiento, cruzando la zona de fractura. Dan cierto grado de estabilidad axial y rotatoria. Con frecuencia

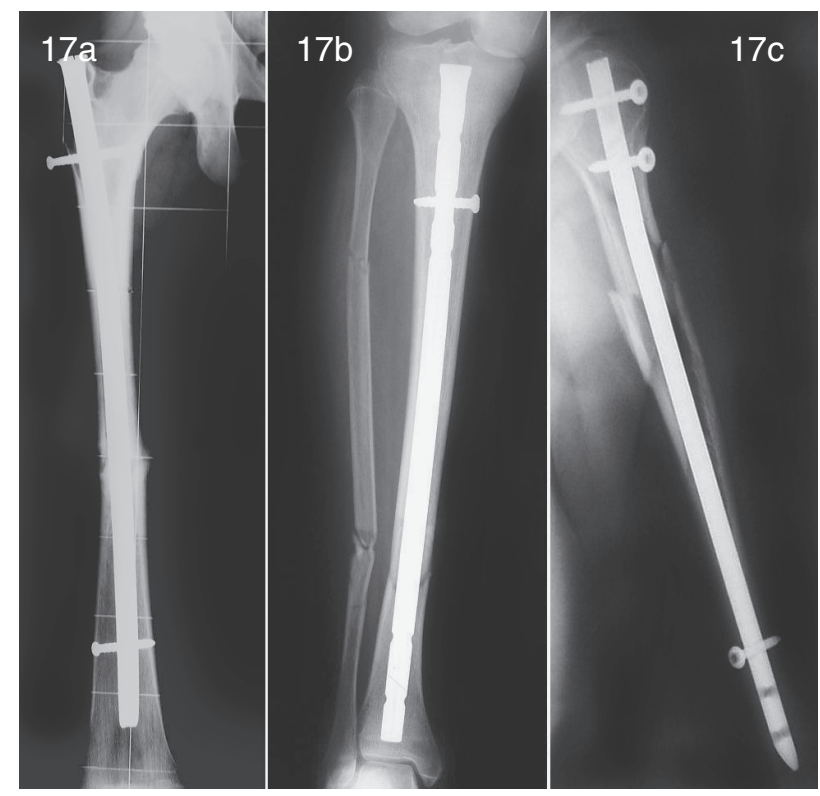

Figura 17 a-c. Clavos intramedulares rígidos: a) Fémur AP. Fractura diafisiaria del tercio medio con bloqueo estático. b) Pierna AP. Fractura de tibia distal con bloqueo dinámico. Además fractura de peroné. c) Húmero AP. Fractura del tercio proximal, con fragmento en mariposa, fijada con clavo intramedular bloqueado proximal y distal. se utilizan con medios de estabilización externa, como yesos. Tienen tasas de infección más bajas que los clavos canulados ${ }^{(1,4)}$ (Figura 19). El clavo Rush, de diámetro pequeño, es biselado en su extremo distal y en forma de gancho en el proximal para facilitar su remoción y evitar la migración. Es utilizado en fracturas del peroné distal y antebrazo. Otro tipo es el clavo de Ender, ligeramente curvo, que también es biselado en su extremo distal(4, 5, 27).

Las complicaciones descritas en los clavos intramedulares incluyen separación de los fragmentos, cambios en la longitud del hueso, penetración articular, inmovilización inadecuada, fallas en el sistema de fijación ${ }^{(1,5,28-29)}$ (Figura 20).

\section{El informe radiológico de fracturas tratadas}

En la práctica diaria nos limitamos a informar "control de tratamiento», "control de consolidación con osteosíntesis metálica», etc. Esto no es un tema menor pues se relaciona con la calidad de la atención global y la información útil entregada a los médicos tratantes, que al no efectuarse nos hace perder credibilidad. Por ende, es de primordial importancia conocer los grupos básicos de elementos de fijación de fracturas, sus características, complicaciones y principios biomecánicos en los que se basa su funcionamiento. Así, se debiera utilizar un lenguaje técnico común con el traumatólogo, tanto desde el punto de vista de los diagnósticos diferenciales como en el seguimiento.

La radiología simple es el método utilizado rutinariamente en el control. Existen algunos

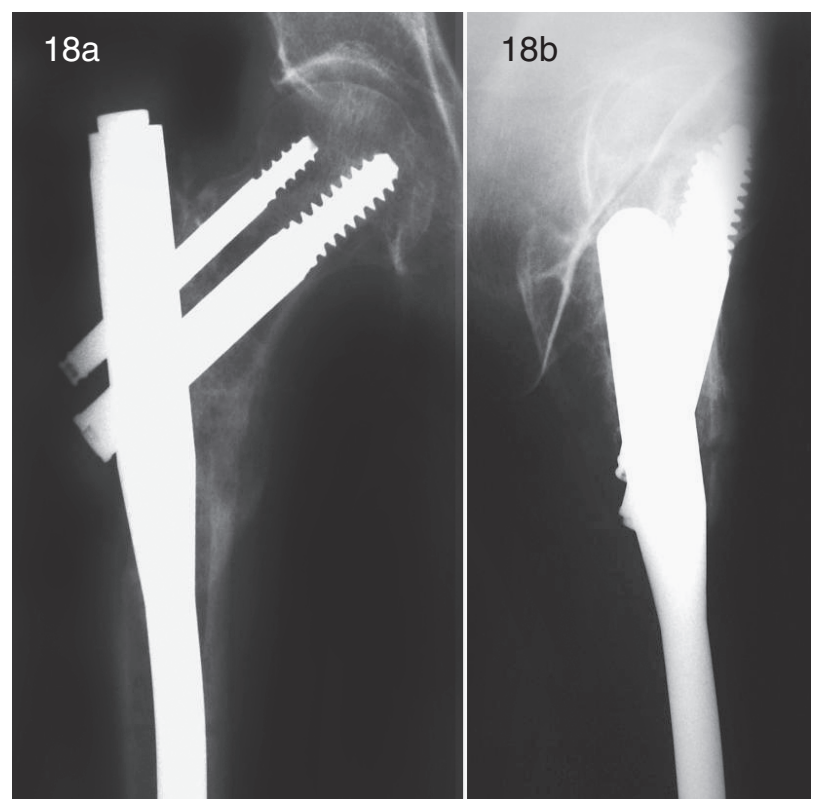

Figura 18 a,b. Clavo de reconstrucción. Cadera AP y L. Fractura subtrocantérea asociada a una fractura diafisiaria (no mostrada). Los agujeros proximales están orientados para que la inserción de los tornillos proximales sea acorde con la anatomía de cabeza y cuello femorales. 
aspectos técnicos básicos a considerar en este tipo de examen, destacándose el obtener como mínimo dos placas ortogonales del sitio de fractura. Si esto resulta difícil por la presencia de implantes metálicos, se obtienen proyecciones oblicuas. Es necesario obtener en hueso largo ambas articulaciones e incluir el elemento de OTS completo en la placa. Una leve sobreexposición es útil para evaluar la fijación metálica, siendo la comparación con los exámenes previos, obligatoria.

En algunos casos se puede requerir de TC multicorte con reconstrucciones en distintos planos, que proporciona información relevante frente a preguntas específicas ¿Hay intrusión articular o en la evaluación de sitios anatómicos complejos como la pelvis? ${ }^{(32-34)}$.

La resonancia magnética se utiliza en el control de las reconstrucciones de ligamento cruzado anterior y en el seguimiento, especialmente cuando se sospechan complicaciones, de las reparaciones con materiales reabsorbibles o biodegradables.

El informe radiológico, una vez que la fractura del paciente ha sido reducida, estabilizada y fijada, cambia desde el polo diagnóstico al del seguimiento con descripción del progreso o no de la cicatrización, y la detección de posibles complicaciones en el hueso o los elementos de OTS. Esto lo podemos resumir en cuatro preguntas básicas: ¿Tipo de fijación? ¿Dónde está? ¿Hay consolidación? ¿Signos de infección? También es importante evaluar la indemnidad de los elementos usados (Figura 20).

Agregamos el Anexo 1 como pauta de recomendación a seguir para un informe adecuado a nuestra consideración.
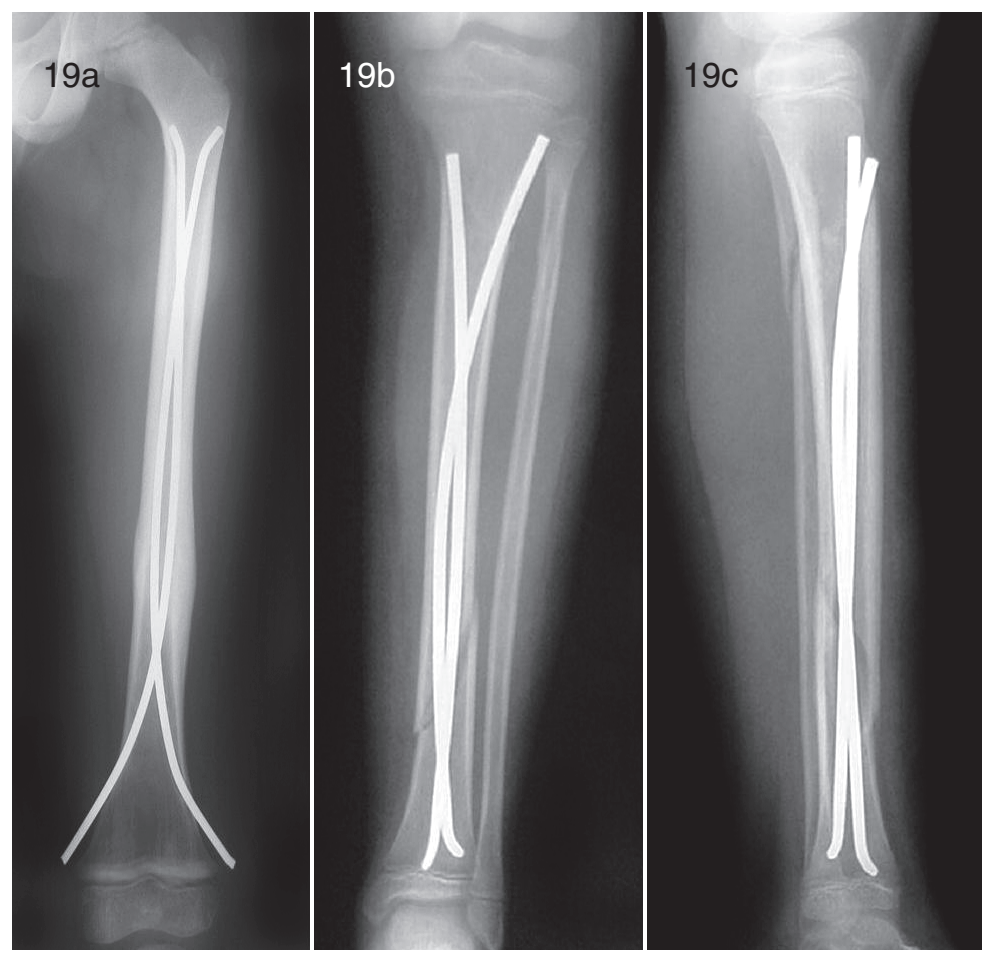

Figura 19 a-c. Clavos intramedulares flexibles: a) Clavo Rush. Esqueleto inmaduro. Fractura del tercio distal del fémur fijada con 2 clavos Rush introducidos en forma anterógrada. b,c) Tibia AP y L. Fractura diafisiaria fijada con 2 clavos Rush introducidos en forma retrógrada. 
Anexo I. Guía para el informe del hueso post-operado

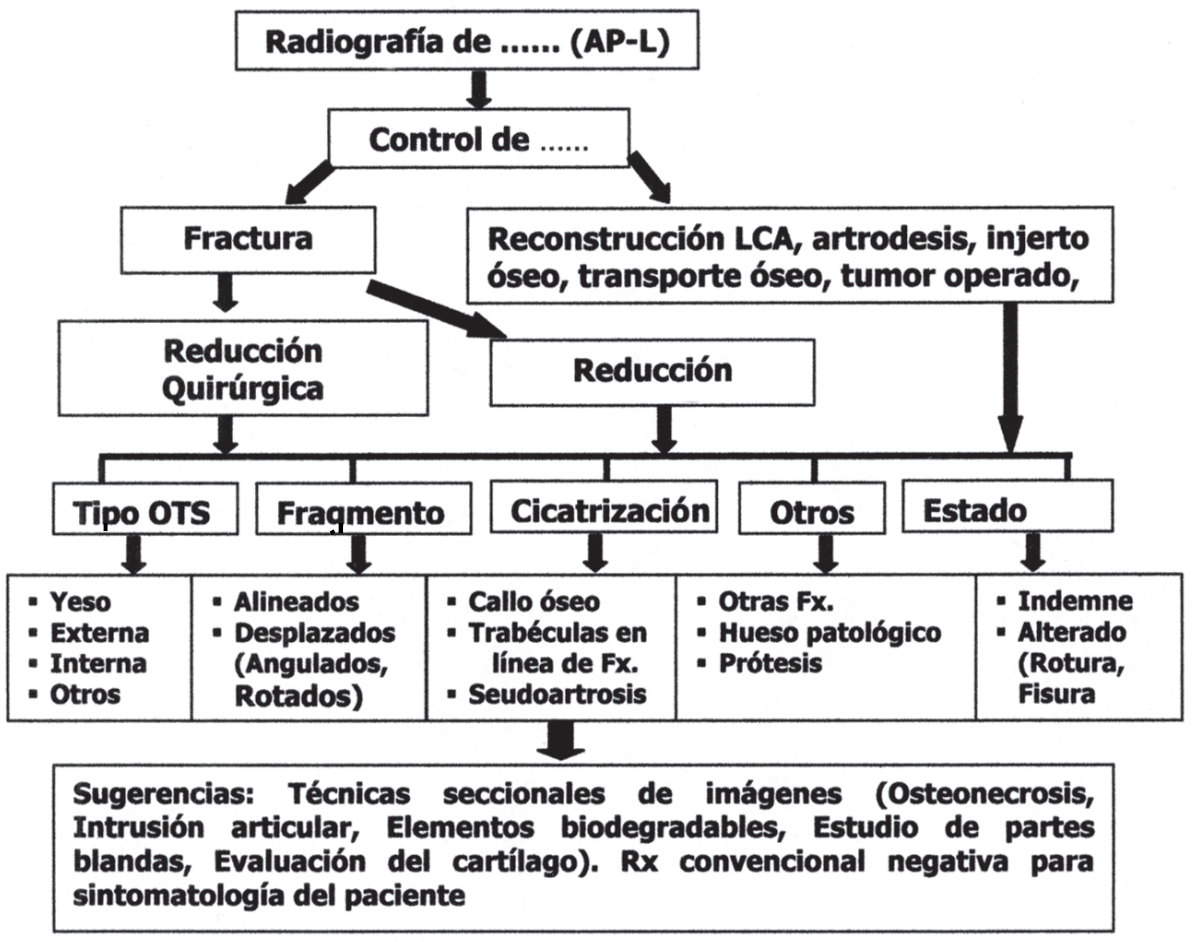

\section{Bibliografía}

1. Chew FS, Pappas CN. Radiology of the devices for fracture. Treatment in the extremities. Clin Radiol North Am 1995; 33: 375-389.

2. Benjamin BB, Lund PJ. Orthopaedic devices. In: Hunter TB, Bragg DG eds. Radiographic guide to medical devices and foreign bodies. St. Louis. Mo: Mosby-Year Book, 1994; 348-385.

3. Althausen PL, Hak DJ. Lower extremity traction pins: Indications, technique, and complications. Am J Orthop 2002; 31: 43-47.

4. Taljanovic MS, Jones MD, Ruth JT, Benjamin JD, Sheppard JE, Hunter TB. Fracture Fixation. RadioGraphics 2003; 23: 1569-1590.

5. Slone RM, Heare MM, Van der Griend RA, Montgomery WJ. Orthopaedic fixation devices. RadioGraphics 1991; 11: 823-847.

6. Gugenheim JJ Jr. External fixation in orthopedics. JAMA 2004; 291: 2122-2124.

7. El Hayek T, Daber AA, Meouchy W, Ley P, Chammas $N$, Griffet J. External fixators in the treatment of fractures in children. J Pediatr Orthop B 2004; 13 : 103-109.

8. Bostman OM. Absorbable implants for fixation of fractures. J Bone Joint Surg Am 1991; 73 A: 148-153.

9. Bechtold JE, Meidt JD, Moed BR,Varecka TF, Bianco
PT. The effect of staple size, orientation number on torsional fractures fixation stability. Clin Orthop 1993; 297:210-217.

10. Herscovici D Jr, Saunders DT, Johnson MP, Sanders R, Di Pasquale T. Percutaneous fixation of proximal humeral fractures. Clin Orthop 2000; 375: 97-104.

11. Manaster BJ, Romley K, Newman AP, Mann FA. Knee ligament reconstruction: plain film analysis. Am J Roentgenol 1998; 150: 337-342.

12. Martin $\mathrm{D}$, Martin TL, Brown $\mathrm{CH}$. Anterior cruciate ligament graft fixation. Orthop Clin North Am 2002; 33: 685-696.

13. Ciccone WJ $2^{\text {nd }}$ Motz C, Tasto JP. Bioabsorbable implants in orthopaedics and clinical applications. J Am Acad Orthop 2001; 9: 280-288.

14. Brand J, Weiler A, Caborn DNM, Brown CH, Johnson DL. Graft Fixation in Cruciate Ligament Reconstruction Am J Sports Med 2000; 28: 761-774.

15. Verhoftad MH, Werken CvC. DHS ostheosyntesis for stable pertrocanteric fractures with four hole side plates. Injury 2004; 35: 999-1002.

16. Chen $\mathrm{CH}$, Chen TB, Cheng YM, Chang JK, Lin SY, Hung SH. Ipsilateral fractures of the femoral neck and shaft. Injury 2000; 31: 719-722.

17. Pai $\mathrm{CH}$. Dynamic condylar screw for subtrochanteric femur fractures with great trochanteric extension. J 
Orthop Trauma 1996; 10: 317-322.

18. Redfern DJ, Syed SU, Davies SJ. Fractures of the distal tibia: minimally invasive plate osteosynthesis Injury 2004; 35: 615-620.

19. Krettek C, Müller M, Miclau T. Evolution of minimally invasive plate osteosynthesis (MIPO) in the femur. Injury 2001; 32 Suppl 3: SC 14-23.

20. Haidukewich GJ. Innovations in Locking Plate Technology. J Am Acad Orthop Surg 2004; 12: 205-212.

21. Tepic S, Perren SM. The biomechanics of the PC-Fix internal fixator. Injury 1995: 26 Suppl:B5-B10.

22. Frigg R, Appenzeller A, Christensen R, Frenk A, Gilbert $\mathrm{S}$, Schavan R. The development of the distal femur. Less Invasive Stabilisation System (LISS). Injury 2001; 32 Suppl 3: SC24- SC31.

23. Syed AA, Agarwal M, Giannoudis PV, Mathews SJ, Smith RM. Distal femoral fractures: long-term outcome following stabilisation with LISS. Injury 2004; 35: 599-607.

24. Larsen LB, Madsen JE, Hoinest P, Ovre S. Should insertion of intramedullary nails for tibial fractures be with or without reaming? A prospective randomised study with 3.8 years' follow up. Orthop Trauma 2004; 18: 144-149.

25. Fernandez FF, Mastschke S, Hulsenbeck A, Egenol FM, Wentzensen A. Five years' clinical experience with the unreamed humeral nail in the treatment of humeral shaft fractures. Injury 2004; 35: 2645-271.
26. Ajmal H, O'Sullivan M, Mc Cabe J, Curtin W. Antegrade locked intramedullary nailing ihumeral shaft fractures. Injury 2001; 32: 692-694.

27. Vrsansky P, Bourdelat D, Al Faour A. Flexible intramedullary pinning in the treatment of paediatrics fractures. J Pediatrics Orthop 2000; 20: 23-27.

28. Pulosky S, Romano C, Buckley R, Powell J. Rotational malalignment of the tibia following reamed intramedullary fixation. J Orthop Trauma 2004; 18: 397-402.

29. Malik MH, Harwood P, Diggle P, Khan SA. Factors affecting rates of infection and non union in the intramedullary fixation. J Bone Joint Surg $\mathrm{Br} 2004 ; 86$ : 556-560.

30. Parikh SN. Bone grafts substitutes in modern orthopedics. Orthopedics 2002;25:1301-311.

31. Ladd AL, Pliam NB. Use of bone graft substitutes in distal radius fracture. J Am Acad Orthop Surg 1999; 7: 279-290.

32. Olsen RV, Munk PL, Lee MF, Fanzen DL, Mackay AL, Xinag Quing-San, Masri B. Metal-Artefact Reduction Sequence: Early Clinical Applications. RadioGraphics 2000; 20: 699-712.

33. White LH, Buckwalter A. Technical Considerations: CT and MR Imaging in the Post-Operative Orthopedics Patient. Seminars in Musculoskeletal Radiology 2002; 6: 5-17.

34. Craig JG, Jacobson JA, Moed BR. Ultrasound of fractures and bone healing. Radiol Clin North Am 1999; 37: 737-751. 